\title{
Quasistationary Probability Density Functions in the Turbulent Mixing of a Scalar Field
}

\author{
Luis Valiño \\ Laboratorio de Investigación en Tecnologías de la Combustión, Consejo Superior de Investigaciones Científicas, \\ M. de Luna 8, Zaragoza 50015, Spain \\ César Dopazo and Javier Ros \\ Universidad de Zaragoza, M. de Luna 3, Zaragoza 50015, Spain
}

(Received 3 November 1993)

\begin{abstract}
The assumption of the existence of an asymptotic shape when $t \rightarrow \infty$ for the probability density function (PDF) of an inert scalar field undergoing turbulent convection and molecular transport [Y. G. Sinai and V. Yakhot, Phys. Rev. Lett. 63, 1962 (1989)], is generalized to include the time evolution. Statistically quasistationary PDFs and conditional scalar dissipation rates compare surprisingly well with direct numerical simulation data at different times. Closed form PDFs are parametrized in terms of the flatness factor solely.
\end{abstract}

PACS numbers: 47.27.--i, 02.50.-r, 05.40.+j

The existence of non-Gaussian limiting probability density functions (PDFs) of fluctuating velocity gradients has for a long time been associated with the internal intermittency of those fields. The non-Gaussianity of scalar fields has recently attracted the attention of both experimentalists [1] and theoreticians [2,3]. The presence of exponential or stretched-exponential tails [4] can be explained via simple approximations once constancy of the normalized statistical moments is assumed to hold after a relaxation time.

The purpose of this Letter is to explore the possible existence of a statistically quasistationary approximate solution valid at all times, allowing the PDF to modify its shape in the process. A dynamically passive temperature field, $T(\boldsymbol{x}, t)$, undergoing turbulent convection and molecular transport obeys the equation

$$
\frac{\partial T}{\partial t}+\boldsymbol{v} \cdot \nabla T=K \nabla^{2} T
$$

where $\boldsymbol{v}(\boldsymbol{x}, t)$ is a solenoidal fluctuating velocity field with zero mean governed by the Navier-Stokes equations and $K$ is the thermal diffusivity. The present Letter is restricted to statistically homogeneous velocity and temperature fields. The time evolution equation for the flatness factor, $F(t)=\left\langle(T-\langle T\rangle)^{4}\right\rangle / \sigma_{T}^{4}$, is

$$
\frac{d F}{d t}=4 F \frac{\left\langle\varepsilon_{T}\right\rangle}{\sigma_{T}^{2}}\left(1-\frac{3}{F} \frac{\left\langle T^{2} \varepsilon_{T}\right\rangle}{\sigma_{T}^{2}\left\langle\varepsilon_{T}\right\rangle}\right)
$$

where $\sigma_{T}^{2}=\left\langle(T-\langle T\rangle)^{2}\right\rangle$ is the fluctuating temperature variance, $\left\langle\varepsilon_{T}\right\rangle=\left\langle K(\nabla T)^{2}\right\rangle$ is the scalar fluctuation dissipation rate, $T^{\prime}=T-\langle T\rangle$ is the temperature fluctuation, and $\langle T\rangle$ is its constant expected value. Sinai and Yakhot [2] observe that $F$ obtained from direct numerical simulation (DNS) [5] reaches a constant value after a period of relaxation. With this simple observation in mind, they extrapolate this asymptotically constant behavior to all even order normalized moments and derive a closed form of the limiting PDF for $t \rightarrow \infty$. However, results for $F(t)$ and for the second term within the parentheses in Eq. (2) from the numerical experiment are shown in Fig. 1 and tend to display an approximate balance of the two terms on the right hand side of (2) over all simulation times. It seems then plausible that a quasistationary PDF, with time as a parameter, may correctly approximate the scalar statistics time evolution. This hypothesis is examined in what follows. The normalized variable, $X(\boldsymbol{x}, t)=T^{\prime}(\boldsymbol{x}, t) / \sigma_{T}(t)$, is governed by

$$
\frac{\partial X}{\partial t}+\boldsymbol{v} \cdot \boldsymbol{\nabla} X=K \nabla^{2} X+\left\langle\varepsilon_{X}\right\rangle X
$$

where $\left\langle\varepsilon_{X}\right\rangle(t)=\left\langle K(\nabla X)^{2}\right\rangle=\left\langle\varepsilon_{T}\right\rangle / \sigma_{T}^{2}$. The finegrained PDF of $X$ is formally

$$
\wp=\delta[\chi-X(\boldsymbol{x}, t)]
$$

and the PDF of $X$ is $P(\chi ; t)=\langle\wp\rangle$. The $P$ transport

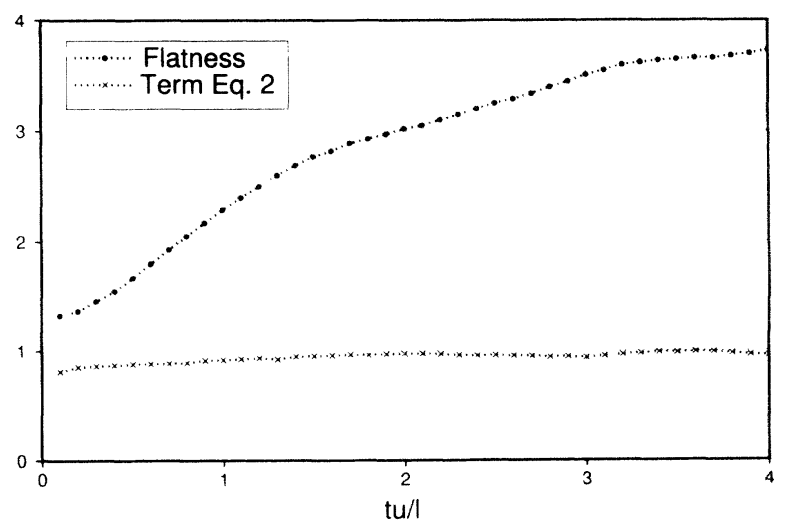

FIG. 1. Evolution of the flatness factor and of the term in Eq. (2) $(3 / F)\left(\left\langle T^{\prime 2} \varepsilon_{T}\right\rangle / \sigma_{T}^{2}\left\langle\varepsilon_{T}\right\rangle\right)$, as a function of the dimensionless time ( $u$ is the turbulence rms velocity and $l$ is the turbulence integral length) obtained from DNS (run F2c [5]). 
equation can take two alternative forms [6],

$$
\begin{gathered}
\frac{\partial P}{\partial t}=-\frac{\partial}{\partial \chi}\left\{\left[\mathfrak{D}(\chi, t)+\left\langle\varepsilon_{X}\right\rangle \chi\right] P\right\}, \\
\frac{\partial P}{\partial t}=-\frac{\partial}{\partial \chi}\left\{\frac{\partial}{\partial \chi}[E(\chi, t) P]+\left\langle\varepsilon_{X}\right\rangle \chi P\right\},
\end{gathered}
$$

where $\mathfrak{D}(\chi, t)=\left\langle K\left(\nabla^{2} X\right) \mid X=\chi\right\rangle$ and $E(\chi, t)=$ $\left\langle K(\nabla X)^{2} \mid X=\chi\right\rangle$ are the conditional diffusion and dissipation rates, respectively. The expressions within the curly brackets in Eqs. (5) are the probability fluxes in the positive direction of the $X$ axis. Identification of the right hand sides of $(5 \mathrm{a})$ and $(5 \mathrm{~b})$ yields

$$
P(\chi, t)=\frac{C_{0}}{E(\chi, t)} \exp \left\{\int_{\chi_{m}}^{\chi} \frac{\mathfrak{D}\left(\chi^{\prime}, t\right)}{E\left(\chi^{\prime}, t\right)} d \chi^{\prime}\right\},
$$

where $C_{0}$ is a normalization constant. Equation (6) can be alternatively obtained averaging the expression for $\nabla^{2} \wp$ and invoking statistical homogeneity. A similar relation for a statistical stationary process is derived averaging $\frac{\partial^{2} \varphi}{\partial t^{2}}[7]$. Equation (6) implies no specific evolution equation.

Multiplication of Eq. (5a) or (5b) by $\chi^{2 n}$ and integration over $\chi$ from $\chi_{m}$ to $\chi_{M}$, the minimum and maximum of $\chi$, respectively, leads to [2]

$$
\frac{d\left\langle X^{2 n}\right\rangle}{d t^{*}}=-2 n(2 n-1) \frac{\left\langle X^{2 n-2} \varepsilon_{X}\right\rangle}{\left\langle\varepsilon_{X}\right\rangle}+2 n\left\langle X^{2 n}\right\rangle,
$$

where $d t^{*}=\left\langle\varepsilon_{X}\right\rangle(t) d t$. The first term on the right hand side of Eq. (7) makes $\left\langle X^{2 n}\right\rangle$ decrease due to diffusive damping, while the second one, which is only a formal consequence of including $\sigma_{T}(t)$ in the definition of the normalized variable $X$, increases $\left\langle X^{2 n}\right\rangle$. An approximate balance between the two terms was assumed by Sinai and Yakhot [2] after a relaxation time. While this balance might be justified using order of magnitude estimates for the velocity gradient [8] and for the scalar gradient, a similar analysis for a scalar field is not a priori easy to apply.

A stationary limit for Eq. (7) also implies that the probability fluxes in Eqs. (5a) and (5b) are independent of $\chi$. In other words, the deflationary and inflationary PDF transport terms in the budget of $X$ fluctuations must approximately be in balance. The dissipation of $T^{\prime}(\boldsymbol{x}, t)$ by molecular diffusion is offset by the simultaneous decay of $\sigma_{T}(t)$ and therefore $T^{\prime}$ is appropriately scaled with $\sigma_{T}$. The characteristic equation in the $\chi-t$ plane corresponding to Eq. (5a) is [6]

$$
\frac{d \chi}{d t}=\mathfrak{D}(\chi, t)+\left\langle\varepsilon_{X}\right\rangle(t) \chi
$$

While $\mathfrak{D}(\chi, t)$ tends to bring any trajectory starting at $\chi=\chi_{0}$ for $t=0$ into the neighborhood of $\chi=0$ (i.e.,
$T=\langle T\rangle)$, the last term in Eq. (8) produces the opposite effect. Quasistationary trajectories thus imply

$$
\mathfrak{D}(\chi, t)=-\left\langle\varepsilon_{X}\right\rangle \chi
$$

which is the linear mean square estimation (LMSE), previously used in turbulent mixing PDF methods [6]. Another characteristic equation equivalent to Eq. (5a) can be integrated resulting in

$$
\begin{aligned}
{\left[\mathfrak{D}(\chi, t)+\left\langle\varepsilon_{X}\right\rangle\right.} & (t) \chi] P(\chi ; t) \\
& =\left[\mathfrak{D}\left(\chi_{0}, 0\right)+\left\langle\varepsilon_{X}\right\rangle(0) \chi_{0}\right] P\left(\chi_{0} ; 0\right) .
\end{aligned}
$$

Quasistationarity of Eq. (5b) leads to

$$
P(\chi ; t)=\frac{C_{1}}{E(\chi, t)} \exp \left[-\left\langle\varepsilon_{X}\right\rangle(t) \int \frac{\chi}{E(\chi, t)} d \chi\right] .
$$

A conservation equation for $E(\chi ; t)$ can be readily derived [9], containing among other terms those describing the strain or rotation of scalar gradients by velocity gradients and the dissipation of $E$ due to molecular diffusive processes. Condensing these turbulent mixing mechanisms into a proposed simple functional dependence of $E$ with $\chi$ has proven not to be a trivial matter. From DNS results [5] and experiments [10], a reasonable polynomial approximation for the conditional dissipation is [2]

$$
\frac{E(\chi, t)}{\left\langle\varepsilon_{X}\right\rangle}=\frac{1+C_{2} \chi^{2}}{1+C_{2}}
$$

should $P(\chi ; t)=P(-\chi ; t)$. Substitution of $(12)$ into (11) and integration yields

$$
P(\chi ; t)=\frac{C_{1}\left(1+C_{2}\right)}{\left\langle\varepsilon_{X}\right\rangle} \frac{1}{\left(1+C_{2} \chi^{2}\right)^{\frac{1+3 C_{2}}{2 C_{2}}}},
$$

which is Sinai and Yakhot's [2] limiting solution for $t \rightarrow \infty$ and $C_{2}>0$. However, in the present context $C_{1}$ and $C_{2}$ must be obtained as functions of the time, $t$, from the normalization condition and moment constraints. DNS [5] and experiments [10] indicate that $C_{2}$ can be either positive or negative. For $C_{2}>0, P(\chi ; t)$ spans over $\chi \in(-\infty,+\infty)$ and for $C_{2}<0, P(\chi ; t)$ is only defined in $\chi \in\left(-\sqrt{-\frac{1}{C_{2}}},+\sqrt{-\frac{1}{C_{2}}}\right)$.

Use of Eq. (7) with the quasistationary approximation $d\left\langle X^{4}\right\rangle / d t^{*}=0$ and of the identity

$$
\left\langle X^{2} \varepsilon_{X}\right\rangle=\int E(\chi, t) \chi^{2} P(\chi ; t) d \chi
$$

allows us to express

$$
C_{2}=\frac{\left\langle X^{4}\right\rangle-3}{2\left\langle X^{4}\right\rangle}
$$

Use of Eqs. (13) and (14) easily yields 


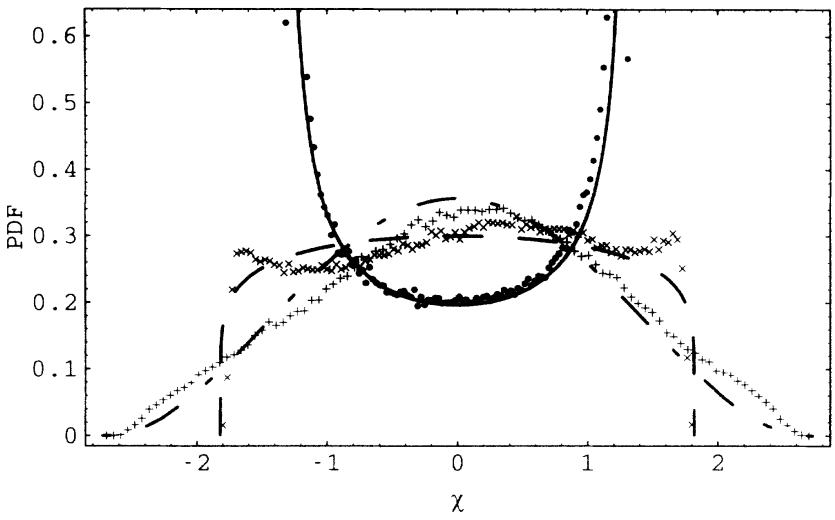

FIG. 2. Comparison of PDFs given by Eqs. (19)-(21) (lines) with those obtained from DNS [11] (inert case: •, $t u / l=0.00 ; \times, t u / l=0.48 ;+, t u / l=0.94)$ at different times.

$$
\frac{C_{1}}{\left\langle\varepsilon_{X}\right\rangle}=\frac{2\left(-C_{2}\right)^{3 / 2}}{1-2 C_{2}} \frac{\Gamma\left(2-\frac{1}{2 C_{2}}\right)}{\sqrt{\pi} \Gamma\left(\frac{1}{2}-\frac{1}{2 C_{2}}\right)}
$$

for $C_{2}<0$,

$$
\frac{C_{1}}{\left\langle\varepsilon_{X}\right\rangle}=\frac{-1}{\sqrt{2 \pi}}
$$

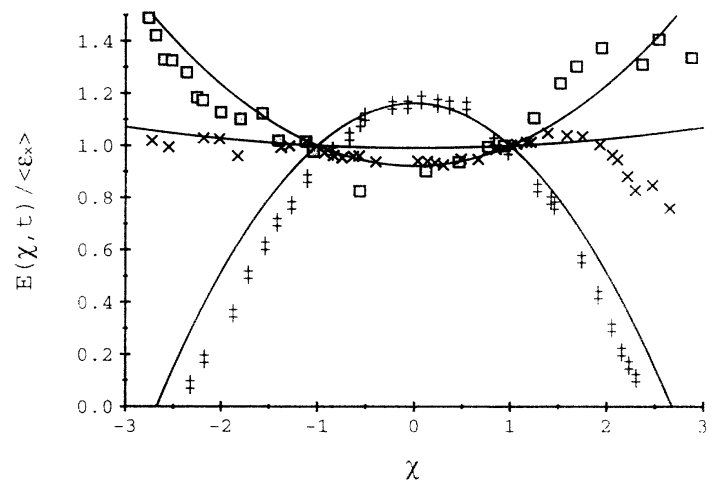

FIG. 3. Comparison of the conditional scalar dissipation rate given by Eq. (12) with $C_{2}$ given by Eq. (15) (lines) and that obtained from DNS [5] (run F2c: $\ddagger, t u / l=1.05 ; \times$, $t u / l=2.11$; gray $\square, t u / l=3.25)$ at different times.

for $C_{2}=0$, and

$$
\frac{C_{1}}{\left\langle\varepsilon_{X}\right\rangle}=\frac{2 C_{2}^{3 / 2}}{1-2 C_{2}} \frac{\Gamma\left(\frac{1}{2}+\frac{1}{2 C_{2}}\right)}{\sqrt{\pi} \Gamma\left(-1+\frac{1}{2 C_{2}}\right)}
$$

for $C_{2}>0$, where $\Gamma(a)$ is the gamma function of the argument $a$. Relations (15) through (18), after setting $\left\langle X^{4}\right\rangle=F$, lead to

$$
P(\chi ; t)=\frac{2}{\sqrt{\pi}}\left(\frac{3-F}{2 F}\right)^{3 / 2} \frac{F-1}{2} \frac{\Gamma\left(\frac{6-F}{3-F}\right)}{\Gamma\left(\frac{3+F}{6-2 F}\right)}\left(1+\frac{F-3}{2 F} \chi^{2}\right)^{\frac{9-5 F}{2(F-3)}}
$$

defined in $\chi \in\left(-\sqrt{\frac{2 F}{3-F}},+\sqrt{\frac{2 F}{3-F}}\right)$ for $1 \leq F<3$, to

$$
P(\chi ; t)=\frac{1}{\sqrt{2 \pi}} \exp \left(\frac{-\chi^{2}}{2}\right)
$$

defined in $\chi \in(-\infty,+\infty)$ for $F=3$, and to

$$
P(\chi ; t)=\frac{2}{\sqrt{\pi}}\left(\frac{F-3}{2 F}\right)^{3 / 2} \frac{F-1}{2} \frac{\Gamma\left(\frac{3 F-3}{2 F-6}\right)}{\Gamma\left(\frac{3}{F-3}\right)}\left(1+\frac{F-3}{2 F} \chi^{2}\right)^{\frac{9-5 F}{2(F-3)}}
$$

defined in $\chi \in(-\infty,+\infty)$ for $F>3$. These PDFs integrate to 1 , have zero odd-order moments, and satisfy the constraints $\left\langle X^{2}\right\rangle=1$ and $\left\langle X^{4}\right\rangle=F$. Figure 2 shows a comparison of the PDFs given by Eqs. (19)(21) and those from DNS results [11]. The agreement is remarkable and well within the numerical experiment error. Figure 3 is a plot of Eq. (12) with $C_{2}$ given by (15) and DNS data [5]. The coincidence is excellent in spite of the poor quality of the DNS predictions near the upper limit of $\chi$. An interesting question to answer in the present context is to what extent initially asymmetric scalar PDFs and/or the addition of a local chemical source term may disrupt the statistical quasistationar- ity assumption. The latter, as examined in this paper, should also be also investigated, for example, for scalar gradient, velocity gradient, and vorticity.

[1] B. Castaing, G. Gunaratne, F. Heslot, L. Kadanoff, A. Libchaber, S. Thomas, X. Z. Wu, S. Zaleski, and G. Zanetti, J. Fluid Mech. 204, 1 (1989).

[2] Ya. G. Sinai and V. Yakhot, Phys. Rev. Lett. 63, 1962 (1989). 
[3] V. Yakhot, S. A. Orszag, S. Balachandar, E. Jackson, Z. S. She, and L. Sirovich, J. Sci. Comput. 5, 199 (1990).

[4] E. S. C. Ching, Phys. Rev. Lett. 70, 283 (1993).

[5] V. Eswaran and S. B. Pope, Phys. Fluids 31, 506 (1988).

[6] C. Dopazo, in Turbulent Reacting Flows, edited by P.A. Libby and F.A. Williams (Academic, New York, 1993).

[7] S. B. Pope and E. S. C. Ching, Phys. Fluids A 5, 1529
(1993).

[8] P. G. Saffman, in Topics in Nonlinear Physics, edited by N. J. Zabusky (Springer-Verlag, New York, 1968).

[9] C. Dopazo and L. Valiño (to be published).

[10] Jayesh and Z. Warhaft, Phys. Fluids A 4, 2292 (1992).

[11] L. Valiño and F. Gao, Phys. Fluids A 4, 2062 (1992). 